\title{
DIMENSION AND HITTING TIME IN RAPIDLY MIXING SYSTEMS
}

\author{
Stefano Galatolo
}

\begin{abstract}
We prove that if a system has superpolynomial (faster than any power law) decay of correlations then the time $\tau_{r}\left(x, x_{0}\right)$ needed for a typical point $x$ to enter for the first time a ball $B\left(x_{0}, r\right)$ centered in $x_{0}$, with small radius $r$ scales as the local dimension at $x_{0}$, i.e.

$$
\lim _{r \rightarrow 0} \frac{\log \tau_{r}\left(x, x_{0}\right)}{-\log r}=d_{\mu}\left(x_{0}\right) .
$$

This result is obtained by proving a kind of dynamical Borel-Cantelli lemma wich holds also in systems having polinomial decay of correlations.
\end{abstract}

\section{Introduction}

Let us consider an ergodic system $(X, T, \mu)$ and two typical points $x, x_{0}$. The orbit of $x$ will come as near as we want to $x_{0}$ entering (sooner or later) in each positive measure neighborhood of $x_{0}$. If $S \subset X$ is such a neighborhood, there is $n \in \mathbb{N}$ such that $T^{n}(x) \in S$. Hitting time (also called waiting time, or shrinking target) problems consider the time

$$
\tau_{S}(x)=\min \left\{n \geq 1: T^{n}(x) \in S\right\}
$$

needed for the orbit of $x$ to enter in $S$ for the first time. As the measure $\mu(S)$ is smaller and smaller, $\tau_{S}(x)$ is bigger and bigger. In systems whose behavior is chaotic enough it can be expected that since (by ergodicity) the orbit of $x$ must visit $S$ with (asymptotic) frequency $\mu(S)$, when $S$ is small, for most $x$

$$
\tau_{S}(x) \sim \frac{1}{\mu(S)} \cdot{ }^{1}
$$

In general systems it is not always like this, even for nice sets, such as balls. There are ergodic systems where the waiting time is generically larger than $\frac{1}{\mu(S)}$. In some sense the behavior of such systems converges "slowly" to the ergodic one.

A way to express this kind of problem in more precise terms is to consider a sequence of balls $B\left(x_{0}, r_{k}\right)$ centered in $x_{0}$ and consider the scaling behavior of the hitting time in such balls, considering as an indicator for the hitting time $R\left(x, x_{0}\right)=$ $\lim _{k \rightarrow \infty} \frac{\log \left(\tau_{B\left(x_{o}, r_{k}\right)}(x)\right)}{-\log \left(r_{k}\right)}$ (hence $\tau_{B\left(x_{o}, r_{k}\right)}(x) \sim r_{k}^{-R\left(x, x_{0}\right)}$ in the sense of scaling behavior equivalence). On the other hand we can consider the scaling behavior of the measure of the balls: $d_{\mu}\left(x_{0}\right)=\lim _{k \rightarrow \infty} \frac{\log \mu\left(B\left(x_{0}, r_{k}\right)\right)}{\log \left(r_{k}\right)}$ (as above this give $\mu\left(B\left(x_{0}, r_{k}\right)\right) \sim r_{k}^{d_{\mu}\left(x_{0}\right)}$ )

\footnotetext{
Received by the editors December 13, 2006.

${ }^{1}$ Here " " stands for some kind of (more or less strict) equivalence in the asymptotic behavior of the two quantities.
} 
this is called the local dimension of $\mu$ at $x_{0}$. It is not difficult to prove that in general systems $R\left(x, x_{0}\right) \geq d_{\mu}\left(x_{0}\right)$ (for precise statements see theorem 2) and there are systems (rotations by irrational angles which are well approximable, see [KS] e.g.) where $\limsup _{k \rightarrow \infty} \frac{\log \left(\mu\left(B\left(x_{0}, r_{k}\right)\right)\right)}{\log \left(r_{k}\right)}>d_{\mu}\left(x_{0}\right)$. Conversely, in many systems with more or less chaotic behavior we have the analog of equation 1 that is

$$
R\left(x, x_{0}\right)=d_{\mu}\left(x_{0}\right)
$$

for $\mu$-almost each $x$. This relation was proved for example for Axiom A systems (and in a weaker form for typical Interval Exchange Transformations, see [G2]), typical rotations on the circle $([\mathrm{KS}])$, systems having exponential distribution of return times ([G], theorem 6) or systems having a strong mixing assumption (called uniform mixing) which is verified in a class of complex dynamical systems (inner functions, see [FMP]).

Hitting time problems are related to many other features of chaotic dynamics: entropy ([Sh]), quantitative recurrence and the distribution of return times (see e.g. $[\mathrm{G}]$, [LHV]), the so called dynamical Borel-Cantelli property (see Definition 5, and references $[\mathrm{GK}]$ or $[\mathrm{FMP}]$ ), asymptotics of Birkhoff sums of functions having infinite average (see [G2]), orbit complexity ([BGI]). Borel-Cantelli properties have also relations with rate of mixing and speed of approximations of points (see e.g. $[\mathrm{T}],[\mathrm{K}]$, $[\mathrm{FMP}])$.

As is well known, in a mixing system we have $\mu\left(A \cap T^{-n}(B)\right) \rightarrow \mu(A) \mu(B)$ for each measurable sets $A, B$. The speed of convergence of the above limit can be arbitrarily slow (depending on $T$ but also on the shape of the sets $A, B$ ). In many systems however the speed of convergence can be estimated for sets having some regularity. Let us remark that considering $1_{A}(x)=\left\{\begin{array}{l}1 \text { if } x \in A \\ 0 \text { if } x \notin A\end{array}\right.$ the mixing condition becomes $\int 1_{B} \circ T^{n} 1_{A} d \mu \rightarrow \int 1_{A} d \mu \int 1_{B} d \mu$. If we consider observables $\phi, \psi: X \rightarrow \mathbb{R}$, in many systems (most of them having some form of hyperbolicity) it can be proved that

$$
\left|\int \phi \circ T^{n} \psi d \mu-\int \phi d \mu \int \psi d \mu\right| \leq\|\phi\|\|\psi\| \Phi(n)
$$

where $\|*\|$ is some Hoelder norm, and $\Phi(n) \rightarrow 0$ is a function whose decay rate is estimated. For example, considering axiom A systems with equilibrium states we have a similar result and $\Phi(n)$ decays exponentially fast. Similar results have been obtained for a large class of more or less hyperbolic systems. The set of references for this kind of results is huge, we cite the books $[B],[\mathrm{V}]$ and $[\mathrm{L}]$, a survey of recent results on nonuniformly expanding systems. Recently fast decay of correlation has also been proved in a large class of systems having strange attractors with dimension close to 1 (Rank 1 systems, see [WY], [WY2]). This class, contains for example the Henon map for some interesting set of parameters.

In this paper we prove (theorem 4) that if the system has a decay of correlations (definition 3) faster than any power law, then for each $x_{0} \in X$ such that $d_{\mu}\left(x_{0}\right)$ exists, eq. 2 holds for almost each $x$. Hence for this kind of systems the typical hitting time scaling rate is given by the local dimension at $x_{0}$.

As a corollary of the proof (corollary 9) we obtain that in systems with fast enough decay of correlations (including some case of polynomial decay) a large class of decreasing sequences of sets have the strong Borel-Cantelli property (see definition 5). 
About this, we remark that in $[\mathrm{T}]$ and $[\mathrm{FMP}]$ other Borel-Cantelli results are obtained supposing different assumptions on the system's mixing behavior (in most cases it is supposed to have some kind of uniform bound on the speed of mixing).

We end by remarking that since eq. 2 is proved for each $x_{0}$ (having local dimension) the hitting time can be used to numerically estimate local dimension at nontypical points. For an example of numerical use of hitting times for this kind of question see $[\mathrm{CG}]$.

\section{Dimension and waiting time}

In the following we will consider a discrete time dynamical system $(X, T)$ where $X$ is a separable metric space equipped with a Borel finite measure $\mu$ and $T: X \rightarrow X$ is a measurable map.

Let us consider the first entrance time of the orbit of $x$ in the ball $B\left(x_{0}, r\right)$ with center $x$ and radius $r$

$$
\tau_{r}\left(x, x_{0}\right)=\min \left(\left\{n \in \mathbf{N}, n>0, T^{n}(x) \in B\left(x_{0}, r\right)\right\}\right) .
$$

By considering the power law behavior of $\tau_{r}\left(x, x_{0}\right)$ as $r \rightarrow 0$ let us define the hitting time indicators as

$$
\bar{R}\left(x, x_{0}\right)=\underset{r \rightarrow 0}{\limsup } \frac{\log \left(\tau_{r}\left(x, x_{0}\right)\right)}{-\log (r)}, \underline{R}\left(x, x_{0}\right)=\liminf _{r \rightarrow 0} \frac{\log \left(\tau_{r}\left(x, x_{0}\right)\right)}{-\log (r)} .
$$

If for some $r, \tau_{r}\left(x, x_{0}\right)$ is not defined then $\bar{R}\left(x, x_{0}\right)$ and $\underline{R}\left(x, x_{0}\right)$ are set to be equal to infinity. The indicators $\bar{R}(x)$ and $\underline{R}(x)$ of quantitative recurrence defined in [BS] are obtained as a special case, $\bar{R}(x)=\bar{R}(x, x), \underline{R}(x)=\underline{R}(x, x)$.

We recall some basic properties of $R\left(x, x_{0}\right)$ which follow from the definition:

Proposition 1. $R\left(x, x_{0}\right)$ satisfies the following properties

- $\bar{R}\left(x, x_{0}\right)=\bar{R}\left(T(x), x_{0}\right), \underline{R}\left(x, x_{0}\right)=\underline{R}\left(T(x), x_{0}\right)$.

- If $T$ is $\alpha$-Hoelder, then $\bar{R}\left(x, x_{0}\right) \geq \alpha \bar{R}\left(x, T\left(x_{0}\right)\right), \underline{R}\left(x, x_{0}\right) \geq \alpha \underline{R}\left(x, T\left(x_{0}\right)\right)$.

Before proceeding and stating connections between hitting time and local dimension, let us recall more precisely some results about dimension. If $X$ is a metric space and $\mu$ is a measure on $X$ the local dimension of $\mu$ at $x$ is defined as $d_{\mu}(x)=\lim _{r \rightarrow 0} \frac{\log (\mu(B(x, r)))}{\log (r)}$ (when the limit exists). Conversely, the upper local dimension at $x \in X$ is defined as $\bar{d}_{\mu}(x)=\limsup _{r \rightarrow 0} \frac{\log (\mu(B(x, r)))}{\log (r)}$ and the lower local dimension $\underline{d}_{\mu}(x)$ is defined in an analogous way by replacing limsup with liminf. If $\bar{d}_{\mu}(x)=\underline{d}_{\mu}(x)=d$ almost everywhere the system is called exact dimensional. In this case many notions of dimension of a measure will coincide. In particular $d$ is equal to the dimension of the measure: $d=\inf \left\{\operatorname{dim}_{H} Z: \mu(Z)=1\right\}$. This happens in a large class of systems. For example in systems having nonzero Lyapunov exponents almost everywhere (see for example the book $[\mathrm{P}]$ ).

In general systems the quantitative recurrence indicator gives only a lower bound on the dimension. The hitting time indicator instead gives an upper bound to the local dimension of the measure at the point $y$. This is summarized in the following 
Theorem 2. ([BGI],[G],[BS], [Bo]) If $(X, T, \mu)$ is a dynamical system over a separable metric space, with an invariant measure $\mu$. For each $x_{0}$

$$
\underline{R}\left(x, x_{0}\right) \geq \underline{d}_{\mu}\left(x_{0}\right), \bar{R}\left(x, x_{0}\right) \geq \bar{d}_{\mu}\left(x_{0}\right)
$$

holds for $\mu$ almost each $x$. Moreover, if $X$ is a closed subset of $\mathbb{R}^{n}$, then for almost each $x \in X$

$$
\bar{R}(x, x) \leq \bar{d}_{\mu}(x), \underline{R}(x, x) \leq \underline{d}_{\mu}(x) .
$$

We remark that eq. 3 implies that if $\alpha<\underline{d}_{\mu}\left(x_{0}\right)$ then

$$
\liminf _{n \rightarrow \infty} n^{\frac{1}{\alpha}} d\left(T^{n}(x), x_{0}\right)=\infty .
$$

In systems with superpolynomial decay of correlations the above inequalities become equalities. For eq. 4 this is proved in $[\mathrm{S}]$. We are going to consider eq. 3. Let us recall more precisely what is superpolynomial decay of correlations.

Definition 3. Let $\phi, \psi: X \rightarrow \mathbb{R}$ be Lipschitz observables on $X$. A system $(X, T, \mu)$ is said to have superpolynomial decay of correlations if

$$
\left|\int \phi \circ T^{n} \psi d \mu-\int \phi d \mu \int \psi d \mu\right| \leq\|\phi\|\|\psi\| \Phi(n)
$$

with $\Phi$ having superpolynolmial decay, i.e. $\lim n^{\alpha} \Phi(n)=0, \forall \alpha>0$. Here \|\| is the Lipschitz norm ${ }^{2}$.

With this definition we can state the main result of the paper:

Theorem 4. If $(X, T, \mu)$ has superpolynomial decay of correlations and $d_{\mu}\left(x_{0}\right)$ exists then

$$
\bar{R}\left(x, x_{0}\right)=\underline{R}\left(x, x_{0}\right)=d_{\mu}\left(x_{0}\right)
$$

for $\mu$-almost each $x$.

We remark that eq. 5 easily implies that if $\alpha>\underline{d}_{\mu}\left(x_{0}\right)$ then

$$
\liminf _{n \rightarrow \infty} n^{\frac{1}{\alpha}} d\left(T^{n}(x), x_{0}\right)=0 .
$$

Before proving theorem 4 we need some lemmas. A sequence of sets $S_{n} \subset X$ is said to be strongly Borel-Cantelli if in some sense the preimages $T^{-n} S_{n}$ cover the space uniformly:

Definition 5. Let $1_{S}$ be the indicator function of the set $S$. The sequence of subsets $S_{n} \subset X$ is said to be a Strongly Borel-Cantelli sequence (SBC) if $\sum_{n} \mu\left(S_{n}\right)=\infty$ and for $\mu$-a.e. $x \in X$ we have as $N \rightarrow \infty$

$$
\frac{\sum_{n=1}^{N} 1_{T^{-n} S_{n}}(x)}{\sum_{n=1}^{N} \mu\left(S_{n}\right)} \rightarrow 1, \mu-\text { a.e. }
$$

\footnotetext{
${ }^{2}$ The case where the Holder norms are considered follows from the case of Lipschitz norm.
} 
We remark that posing $Z_{k}(x)=\sum_{0}^{k} 1_{T^{-i} S_{i}}(x)$ the above condition is equivalent to

$$
\frac{Z_{k}}{E\left(Z_{k}\right)} \rightarrow 1, \mu-\text { a.e. }
$$

The following technical lemma estimates the speed of mixing of balls in systems having some given decay of correlations.

Lemma 6. Let $B\left(x_{0}, r_{k}\right)$ be a sequence of balls with decreasing radius centered in $x_{0}$, let $A_{k}=T^{-k}\left(B\left(x_{0}, r_{k}\right)\right)$ and let us write $A_{-1}=X$. If $(X, T, \mu)$ is a system satisfying definition 3 then when $k>j>0$

$$
\mu\left(A_{k} \cap A_{j}\right) \leq \mu\left(A_{k-1}\right) \mu\left(A_{j-1}\right)+\frac{\Phi(k-j)}{\left(r_{k-1}-r_{k}\right)\left(r_{j-1}-r_{j}\right)} .
$$

Proof. Let $\phi_{k}$ be a Lipschitz function such that $\phi_{k}(x)=1$ for all $x \in B\left(x_{0}, r_{k}\right)$, $\phi_{k}(x)=0$ if $x \notin B\left(x_{0}, r_{k-1}\right)$ and $\left\|\phi_{k}\right\| \leq \frac{1}{r_{k-1}-r_{k}}$ (such functions can be easily constructed as $\phi_{k}(x)=h\left(d\left(x_{0}, x\right)\right)$ where $h$ is a suitable piecewise linear Lipschitz function $\mathbb{R} \rightarrow[0,1])$. Let $k>j>0$. Since $\mu$ is preserved

$$
\begin{gathered}
\mu\left(A_{k} \cap A_{j}\right)=\mu\left(T^{-k+j}\left(B\left(x_{0}, r_{k}\right)\right) \cap B\left(x_{0}, r_{j}\right)\right) \leq \int \phi_{k} \circ T^{k-j} \phi_{j} d \mu \leq \\
\leq \int \phi_{k} d \mu \int \phi_{j} d \mu+\left\|\phi_{k}\right\|\left\|\phi_{j}\right\| \Phi(k-j) \leq \mu\left(A_{k-1}\right) \mu\left(A_{j-1}\right)+\left\|\phi_{k}\right\|\left\|\phi_{j}\right\| \Phi(k-j)
\end{gathered}
$$

which gives the statement.

In the following we will prove that if a decreasing sequence of sets $S_{k}$ are rapidly mixed like in eq. 6 , then $T^{-k}\left(S_{k}\right)$ covers $X$ in a uniform way, i.e. the $S_{k}$ form a SBC sequence. The idea of the proof of this is somewhat similar to the proof of the strong law of large numbers using the Paley-Zygmund inequality, and to the proof of theorem 1 in [FMP]. The idea is to estimate $E\left(\left(Z_{n}\right)^{2}\right)$ (this will be done by using something similar to eq. 6) and find an upper bound which ensures that the distribution of the possible values of $Z_{n}$ is not too far from the average $E\left(Z_{n}\right)$. After this, choosing suitable subsequences we prove also pointwise convergence, so that $\frac{Z_{k}}{E\left(Z_{k}\right)} \rightarrow 1, \mu-a . e$.

Lemma 7. Let $S_{k}$ be a decreasing sequence of measurable sets such that

$$
\liminf _{k \rightarrow \infty} \frac{\log \left(\sum_{0}^{k} \mu\left(S_{k}\right)\right)}{\log (k)}=z>0 .
$$

Let $A_{k}=T^{-k}\left(S_{k}\right)$ and let us suppose that the system is such that when $k>j$

$$
\mu\left(A_{k} \cap A_{j}\right) \leq \mu\left(A_{k-1}\right) \mu\left(A_{j-1}\right)+k^{c_{1}} j^{c_{2}} \Phi(k-j)
$$

with $\Phi$ having superpolynomial decay and $c_{1}, c_{2} \geq 0$. Then posing $Z_{k}(x)=\sum_{0}^{k} 1_{A_{i}}(x)$ we have $\frac{Z_{k}}{E\left(Z_{k}\right)} \rightarrow 1$ in the $L^{2}$ norm and almost everywhere.

Proof. Let us estimate $E\left(\left(Z_{n}\right)^{2}\right)$. We have that

$$
E\left(\left(Z_{n}\right)^{2}\right)=\sum_{k=1}^{n} \mu\left(A_{k}\right)+2 \sum_{k, j \leq n, k>j} \mu\left(A_{k} \cap A_{j}\right) .
$$


Now let $0<\alpha<\frac{z}{2}$ and let us estimate the second summand on the right side by dividing it into two parts in the following way

$$
\sum_{k, j \leq n, k>j} \mu\left(A_{k} \cap A_{j}\right) \leq \sum_{k, j \leq n, k>j, k<j+n^{\alpha}} \mu\left(A_{k} \cap A_{j}\right)+\sum_{k, j \leq n, k \geq j+n^{\alpha}} \mu\left(A_{k} \cap A_{j}\right) .
$$

The first sum can be estimated as follows:

$$
\sum_{k, j \leq n, k>j, k<j+n^{\alpha}} \mu\left(A_{k} \cap A_{j}\right) \leq n^{\alpha} E\left(Z_{n}\right) .
$$

In the second one we use equation 7 and we obtain

$$
\begin{gathered}
\sum_{k, j \leq n, k \geq j+n^{\alpha}} \mu\left(A_{k} \cap A_{j}\right) \leq \sum_{k, j \leq n, k \geq j+n^{\alpha}} \mu\left(A_{k-1}\right) \mu\left(A_{j-1}\right)+n^{c_{1}+c_{2}} \Phi\left(n^{\alpha}\right) \leq \\
\leq \frac{1}{2}\left(E\left(Z_{n}\right)\right)^{2}+n^{2+c_{1}+c_{2}} \Phi\left(n^{\alpha}\right) .
\end{gathered}
$$

Hence

$$
\sum_{k, j \leq n, k>j} \mu\left(A_{k} \cap A_{j}\right) \leq n^{\alpha} E\left(Z_{n}\right)+\frac{1}{2}\left(E\left(Z_{n}\right)\right)^{2}+n^{2+c_{1}+c_{2}} \Phi\left(n^{\alpha}\right)
$$

and

$$
E\left(\left(Z_{n}\right)^{2}\right) \leq\left(2 n^{\alpha}+1\right) E\left(Z_{n}\right)+\left(E\left(Z_{n}\right)\right)^{2}+2 n^{2+c_{1}+c_{2}} \Phi\left(n^{\alpha}\right) .
$$

Now, let us remark that

$$
E\left(\left(Z_{n}-E\left(Z_{n}\right)\right)^{2}\right)=E\left(\left(Z_{n}\right)^{2}\right)-\left(E\left(Z_{n}\right)\right)^{2}
$$

hence

$$
E\left(\left(Z_{n}-E\left(Z_{n}\right)\right)^{2}\right) \leq\left(2 n^{\alpha}+1\right) E\left(Z_{n}\right)+2 n^{2+c_{1}+c_{2}} \Phi\left(n^{\alpha}\right) .
$$

Now we want to compare $Z_{n}$ with its average $E\left(Z_{n}\right)$. For this we consider

$$
Y_{n}=\frac{Z_{n}}{E\left(Z_{n}\right)}-1=\frac{Z_{n}-E\left(Z_{n}\right)}{E\left(Z_{n}\right)} .
$$

When $Y_{n}=0, Z_{n}=E\left(Z_{n}\right)$. By the above results

$$
E\left(\left(Y_{n}\right)^{2}\right) \leq \frac{\left(2 n^{\alpha}+1\right) E\left(Z_{n}\right)+2 n^{2+c_{1}+c_{2}} \Phi\left(n^{\alpha}\right)}{\left(E\left(Z_{n}\right)\right)^{2}}
$$

since $\alpha<\frac{z}{2}$ and $2 n^{2+c_{1}+c_{2}} \Phi\left(n^{\alpha}\right) \rightarrow 0$, then $\lim _{n \rightarrow \infty} E\left(\left(Y_{n}\right)^{2}\right)=0$. This proves that $\frac{Z_{n}}{E\left(Z_{n}\right)} \rightarrow 1$ in $L^{2}$.

By this it is easy to see that there is a subsequence of $Y_{n}$ that converges a.e. to 0 , but we want to prove that the whole sequence converges to 0 a.e. We begin by considering

$$
n_{k}=\inf \left\{n: E\left(Z_{n}\right) \geq k^{2}\right\}
$$

and show that $Y_{n_{k}} \rightarrow 0$ a.e. We remark that since $\Phi$ has a superpolynomial decay

$$
\sum_{n} n^{2+c_{1}+c_{2}} \frac{\Phi\left(n^{\alpha}\right)}{\left(E\left(Z_{n}\right)\right)^{2}}<\infty .
$$

Consider a small $\epsilon>0$, by definition of $z$ and by the fact that $\mu\left(A_{i}\right)<1$ we have that if $k$ is big enough $(k+1)^{2} \geq E\left(Z_{n_{k}}\right) \geq\left(n_{k}\right)^{z-\epsilon}$. Hence $n_{k} \leq(k+1)^{\frac{2}{z-\epsilon}} \leq(2 k)^{\frac{2}{z-\epsilon}}$ 
and so $\frac{\left(2 n_{k}^{\alpha}+1\right) E\left(Z_{n_{k}}\right)}{\left(E\left(Z_{n_{k}}\right)\right)^{2}}=\frac{2 n_{k}^{\alpha}+1}{E\left(Z_{n_{k}}\right)} \leq \frac{2(2 k)^{\frac{2 \alpha}{z-\epsilon}}+1}{k^{2}}$. Since $\alpha<\frac{z}{2}$, we can suppose $\epsilon$ to be so small that $2 \alpha<z-\epsilon$ and hence

$$
\frac{2 \alpha}{z-\epsilon}-2<-1
$$

This implies that $\sum E\left(\left(Y_{n_{k}}\right)^{2}\right)<\infty$, which in turn implies that $Y_{n_{k}} \rightarrow 0$ a.e. and $\frac{Z_{n_{k}}}{E\left(Z_{n_{k}}\right)} \rightarrow 1$ almost everywhere.

Now, if $n_{k} \leq n \leq n_{k+1}$

$$
\frac{Z_{n}}{E\left(Z_{n}\right)} \leq \frac{Z_{n_{k+1}}}{E\left(Z_{n_{k}}\right)}=\frac{Z_{n_{k+1}}}{E\left(Z_{n_{k+1}}\right)} \frac{E\left(Z_{n_{k+1}}\right)}{E\left(Z_{n_{k}}\right)} \leq \frac{Z_{n_{k+1}}}{E\left(Z_{n_{k+1}}\right)} \frac{(k+2)^{2}}{k^{2}}
$$

and

$$
\frac{Z_{n}}{E\left(Z_{n}\right)} \geq \frac{Z_{n_{k}}}{E\left(Z_{n_{k+1}}\right)}=\frac{Z_{n_{k}}}{E\left(Z_{n_{k}}\right)} \frac{E\left(Z_{n_{k}}\right)}{E\left(Z_{n_{k+1}}\right)} \geq \frac{Z_{n_{k}}}{E\left(Z_{n_{k}}\right)} \frac{k^{2}}{(k+2)^{2}} .
$$

then we have $\lim _{n \rightarrow \infty} \frac{Z_{n}}{E\left(Z_{n}\right)}=1, \mu$-almost everywhere.

Remark 8. We remark that in the above proof, the key point to ensure that $\sum E\left(\left(Y_{n_{k}}\right)^{2}\right)<\infty$ (and then have a.e. convergence) are equations 12 and 13 this implies that if $\Phi$ has not superpolinomial decay, but a polinomial decay fast enough that $\sum_{n} n^{2+c_{1}+c_{2}} \frac{\Phi\left(n^{\alpha}\right)}{\left(E\left(Z_{n}\right)\right)^{2}}<\infty$ for some $\alpha<\frac{z}{2}$ then $\lim _{n \rightarrow \infty} \frac{Z_{n}}{E\left(Z_{n}\right)}=1$, $\mu$-almost everywhere. Then the lemma also holds for some system such that $\Phi$ has (rapid enough) polinomial decay.

Now we use the above result to conclude the equality between the hitting time indicator and dimension. The second part of the proof is similar to the proof of Theorem 2.4 in $[\mathrm{GK}]$.

Proof. (of Thm. 4) Let us prove $\bar{R}\left(x, x_{0}\right) \leq d_{\mu}\left(x_{0}\right)$ for almost each $x$. We recall that this implies $\underline{R}\left(x, x_{0}\right) \leq d_{\mu}\left(x_{0}\right)$ and the opposite inequalities come from theorem 2. Let us consider $0<\beta<\frac{1}{d_{\mu}\left(x_{0}\right)}$ and a sequence $r_{k}=k^{-\beta}$ (we remark that if the result is proved for such a subsequence, hence it holds for all subsequences, see lemma 4.2 in $[\mathrm{GKP}])$. Then for each small $\epsilon<\beta^{-1}-d_{\mu}\left(x_{0}\right)$, eventually $\mu\left(B\left(x_{0}, r_{k}\right)\right) \geq$ $\left(r_{k}\right)^{d_{\mu}\left(x_{0}\right)+\epsilon}=k^{-\beta\left(d_{\mu}\left(x_{0}\right)+\epsilon\right)}$ and if $k$ is big enough $\sum_{0}^{k} \mu\left(B\left(x_{0}, r_{i}\right)\right) \geq C k^{1-\beta\left(d_{\mu}\left(x_{0}\right)+\epsilon\right)}$, since $\epsilon$ is arbitrary we have

$$
\liminf _{k \rightarrow \infty} \frac{\log \left(\sum_{0}^{k} \mu\left(B\left(x_{0}, r_{i}\right)\right)\right)}{\log (k)} \geq 1-\beta d_{\mu}\left(x_{0}\right)>0 .
$$

Moreover, $r_{k-1}-r_{k} \sim k^{-\beta-1}$. Hence we can apply Lemma 6 and 7 to the sequence $B\left(x_{0}, r_{k}\right)$ and obtain that for such a sequence $\lim _{n \rightarrow \infty} \frac{Z_{n}}{E\left(Z_{n}\right)}=1, \mu$-almost everywhere.

Let us consider $\epsilon^{\prime}>0$ and $\beta$ as above, near to $\frac{1}{d_{\mu}\left(x_{0}\right)}$, such that $\beta\left(d_{\mu}\left(x_{0}\right)+\epsilon^{\prime}\right)>1$. Moreover let us consider $\varepsilon>0$ so small that $\beta\left(d_{\mu}\left(x_{0}\right)+\varepsilon\right)<1$ and $\beta\left(d_{\mu}\left(x_{0}\right)+\right.$ $\left.\epsilon^{\prime}\right)-\frac{1-\beta\left(d_{\mu}\left(x_{0}\right)-\varepsilon\right)}{1-\beta\left(d_{\mu}\left(x_{0}\right)+\varepsilon\right)}>0$. Let us consider $x$ such that $\bar{R}\left(x, x_{0}\right)>d_{\mu}\left(x_{0}\right)+\epsilon^{\prime}$, then for infinitely many $n, \tau_{n^{-\beta}}\left(x, x_{0}\right)>n^{\beta\left(d_{\mu}\left(x_{0}\right)+\epsilon^{\prime}\right)}$. Then

$$
x \notin \cup_{0 \leq i \leq n^{\beta\left(d_{\mu}\left(x_{0}\right)+\epsilon^{\prime}\right)}} T^{-i}\left(B\left(x_{0}, n^{-\beta}\right)\right)
$$


and in particular

$$
x \notin \cup_{n \leq i \leq n^{\beta\left(d_{\mu}\left(x_{0}\right)+\epsilon^{\prime}\right)}} T^{-i}\left(B\left(x_{0}, n^{-\beta}\right)\right) \supset \cup_{n \leq i \leq n^{\beta\left(d_{\mu}\left(x_{0}\right)+\epsilon^{\prime}\right)}} T^{-i}\left(B\left(x_{0}, i^{-\beta}\right)\right),
$$

which implies that there is a sequence $n_{i}$ such that $Z_{n_{i}}(x)=Z_{n_{i}^{\beta\left(d_{\mu}\left(x_{0}\right)+\epsilon^{\prime}\right)}}(x)$ for each

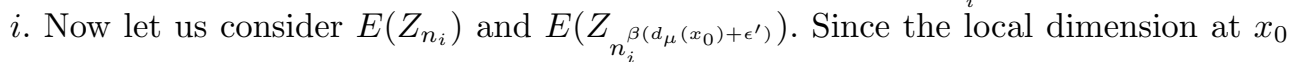
is $d_{\mu}\left(x_{0}\right)$, when $i$ is big enough

$$
i^{-\beta\left(d_{\mu}\left(x_{0}\right)+\varepsilon\right)}<\mu\left(B\left(x_{0}, i^{-\beta}\right)\right)<i^{-\beta\left(d_{\mu}\left(x_{0}\right)-\varepsilon\right)}
$$

then there are constants $k_{1}$ and $k_{2}$ such that when $n$ is big enough $k_{1} n^{1-\beta\left(d_{\mu}\left(x_{0}\right)+\varepsilon\right)}<$ $E\left(Z_{n}\right)<k_{2} n^{1-\beta\left(d_{\mu}\left(x_{0}\right)-\varepsilon\right)}$. From this we have that if $i$ is big enough

$$
\begin{aligned}
& \frac{E\left(Z_{n_{i}}\right)}{E\left(Z_{\left.n_{i}^{\beta\left(d_{\mu}\left(x_{0}\right)+\epsilon^{\prime}\right)}\right)}\right.} \leq \frac{k_{2} n_{i}^{1-\beta\left(d_{\mu}\left(x_{0}\right)-\varepsilon\right)}}{k_{1} n_{i}^{\beta\left(d_{\mu}\left(x_{0}\right)+\epsilon^{\prime}\right)\left(1-\beta\left(d_{\mu}\left(x_{0}\right)+\varepsilon\right)\right)}}= \\
& =\frac{k_{2}}{k_{1}} n_{i}^{\left(1-\beta\left(d_{\mu}\left(x_{0}\right)-\varepsilon\right)\right)-\beta\left(d_{\mu}\left(x_{0}\right)+\epsilon^{\prime}\right)\left(1-\beta\left(d_{\mu}\left(x_{0}\right)+\varepsilon\right)\right)} .
\end{aligned}
$$

By the assumptions on $\varepsilon,\left(1-\beta\left(d_{\mu}\left(x_{0}\right)-\varepsilon\right)\right)-\beta\left(d_{\mu}\left(x_{0}\right)+\epsilon^{\prime}\right)\left(1-\beta\left(d_{\mu}\left(x_{0}\right)+\varepsilon\right)\right)=$ $\left(1-\beta\left(d_{\mu}\left(x_{0}\right)+\varepsilon\right)\right)\left(\frac{1-\beta\left(d_{\mu}\left(x_{0}\right)-\varepsilon\right)}{1-\beta\left(d_{\mu}\left(x_{0}\right)+\varepsilon\right)}-\beta\left(d_{\mu}\left(x_{0}\right)+\epsilon^{\prime}\right)\right)<0$, hence

$$
\lim _{i \rightarrow \infty} \frac{E\left(Z_{n_{i}}\right)}{E\left(Z_{\left.n_{i}^{\beta\left(d_{\mu}\left(x_{0}\right)+\epsilon^{\prime}\right)}\right)}\right.}=0 .
$$

Since $n_{i}$ was chosen such that $Z_{n_{i}}(x)=Z_{n_{i}^{\beta\left(\bar{d}_{\mu}\left(x_{0}\right)+\epsilon^{\prime}\right)}}(x)$ this implies that

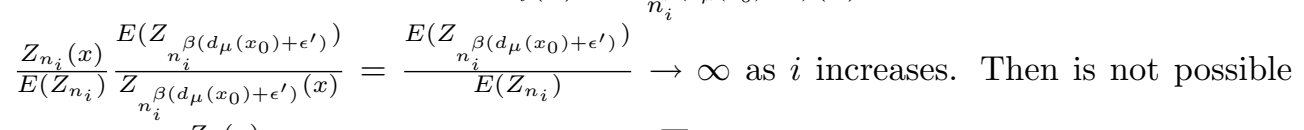
that $\lim _{n \rightarrow \infty} \frac{Z_{n}(x)}{E\left(Z_{n}(x)\right)}=1$. This, implies that $\bar{R}\left(x, x_{0}\right)>d_{\mu}\left(x_{0}\right)+\epsilon^{\prime}$ on a zero measure set. Finally, since $\epsilon^{\prime}$ can be chosen to be arbitrarily small we have the statement.

We end by remarking that the above lemmas 6,7 and remark 8 give the following consequence, which in the author opinion is interesting by itself (by lemma 7, the proof is obtained as in the first part of the proof of thm. 4):

Corollary 9. If the system has decay of correlation given by the function $\Phi$ (see definition 3) and $B\left(x_{0}, r_{i}\right)$ is a sequence of decreasing balls such that

i) $\liminf _{k \rightarrow \infty} \frac{\log \left(\sum_{0}^{k} \mu\left(B\left(x_{0}, r_{i}\right)\right)\right)}{\log (k)}=z>0$,

ii) $\liminf _{n \rightarrow \infty} \frac{\log \left(r_{n-1}-r_{n}\right)}{\log (n)}=c \in \mathbb{R}$

iii) $\sum_{n} n^{2-2 c+\epsilon} \frac{\Phi\left(n^{\alpha}\right)}{\left(\sum_{0}^{k} \mu\left(B\left(x_{0}, r_{i}\right)\right)\right)^{2}}<\infty$ for some $\alpha<\frac{z}{2}$ and $\epsilon>0$

then $B\left(x_{0}, r_{i}\right)$ has the $S B C$ property.

\section{References}

[B] V. Baladi Positive transfer operators and decay of correlations, Adv. Ser. Nonlin. Anal. v.16 World Scientific, River Edge, 2000.

[BS] L. Barreira and B. Saussol, Hausdorff dimension of measures via Poincaré recurrence, Commun. Math. Phys., 219 (2001), 443-463. 
[BGI] C. Bonanno, S. Isola, and S. Galatolo, Recurrence and algorithmic information, Nonlinearity 17 (2004), no. 3, 1057-1074.

[Bo] M. D. Boshernitzan, Quantitative recurrence results, Invent. Math. 113 (1993), 617-631.

[FMP] J. L. Fernandez, M. .V. Melian, and D. Pestana, Quantitative mixing results and inner functions, Math. Ann 337 (2007), no. 1, 233-251.

[CG] T. Carletti and S. Galatolo, Numerical Estimates of dimension by recurrence and waiting time, Physica A (2006). 81-587.

[G] S. Galatolo, . Dimension via waiting time and recurrence, Math. Res. Lett. 12, no 3, May 2005, 377-386.

[G2] _ Hitting time and dimension in axiom A systems, generic interval exchanges and an application to Birkoff sums. J. Stat. Phys. 123 (2006), no. 1, 111-124.

[GK] S. Galatolo and D. H. Kim, The dynamical Borel-Cantelli lemma and the waiting time problems. Preprint, Arxiv: math.DS/0610213.

[GKP] S. Galatolo, D. H. Kim, and K. Park, The recurrence time for ergodic systems with infinite invariant measures, Nonlinearity 19 No 11 (November 2006) 2567-2580

[KS] D. H. Kim and B. K. Seo, The waiting time for irrational rotations, Nonlinearity 16 (2003), no. $5,1861-1868$.

[K] D. Kleinbock, Metric Diophantine approximation and dynamical systems, Lecture notes, Brandeis University, http://people.brandeis.edu/ kleinboc/203b/lectures.pdf.

[L] S. Luzzatto, Stochastic-like behaviour in non-uniformly expanding maps, Handbook of Dynamical Systems, Vol. 1B, 265-326 B. Hasselblatt and A. Katok (Eds), Elsevier. (2006).

[LHV] Y. Lacroix, N. Haydn, and S. Vaienti, Hitting and return times in ergodic dynamical systems, Ann. Probab. 33 (2005), no. 5, 2043-2050.

[P] Y. Pesin, Dimension theory in dynamical systems, Chicago lectures in Mathematics (1997).

[S] B. Saussol, Recurrence rate in rapidly mixing dynamical systems. Discrete Contin. Dyn. Syst. 15 (2006), no. 1, 259-267.

[Sh] P.C. Shields, The ergodic theory of discrete sample paths, Graduate Studies in Mathematics 13, American Mathematical Society, Providence, RI, 1996.

[T] D. Tasche, On the second Borel-Cantelli lemma for strongly mixing sequences of events. J. Appl. Probab. 34 (1997), no. 2, 381-394.

[V] M. Viana, Stochastic dynamics of deterministic systems, Lecture notes, http://w3.impa.br/ ${ }^{\sim}$ viana/out/sdds.pdf.

[WY] Q. Wang and L. S. Young, Strange attractors with one direction of instability, Comm. Math. Phis. 218 (2001), no. 1, 1-97.

[WY2] _ , Towards a theory of rank one attractors, to appear on Ann. Math.

Dipartimento di Matematica Applicata, Universita di Pisa, Via Buonarroti 1, Pisa

E-mail address: s.galatolo@ing.unipi.it 